\title{
The burden of insomnia in Japan
}

\author{
This article was published in the following Dove Press journal: \\ Nature and Science of Sleep \\ 5 January 2015 \\ Number of times this article has been viewed
}

\section{Kazuo Mishima' \\ Marco daCosta \\ DiBonaventura ${ }^{2}$ \\ Hillary Gross ${ }^{2}$}

'Department of Psychophysiology, National Institute of Mental Health, National Center of Neurology and Psychiatry, Kodaira, Tokyo, Japan; ${ }^{2}$ Kantar Health, New York, NY, USA

Correspondence: Marco daCosta

DiBonaventura

Kantar Health, II Madison Avenue,

12th Floor, New York, NY 10010, USA

$\mathrm{Tel}+$ I 2127063988

Fax + I 2126477659

Email marco.dibonaventura@

kantarhealth.com
Objectives: Several studies have suggested that patients who experience insomnia report a number of significant impairments. However, despite this literature, fewer studies have focused on the burden of insomnia among patients in Japan. The objective of the current study is to extend this work in Japan to further understand the effect of insomnia on health-related quality of life (hrQOL). Further, another objective is to understand general predictors of hrQOL among patients with insomnia.

Methods: Data from the 2012 Japan National Health and Wellness Survey, an annual, crosssectional study of adults aged 18 years or older, were used $(\mathrm{N}=30,000)$. All National Health and Wellness Survey respondents were categorized based on the incidence of self-reported insomnia diagnosis and prescription medication usage (clinical insomniacs under treatment versus [vs] good sleepers without insomnia or insomnia symptoms). Comparisons among different groups were made using multiple regression models controlling for demographics and health history.

Results: Clinical insomniacs $(n=1,018 ; 3.4 \%)$ reported significantly worse hrQOL compared with good sleepers $(n=20,542)$ (mental component summary: 34.2 vs 48.0 ; physical component summary: 48.0 vs 52.8 ; health utilities: 0.61 vs 0.76 ; all $P<0.05$ ). Health behaviors (smoking, exercise, alcohol use) and comorbidities were the strongest predictors of health utilities for clinical insomniacs. For all three clinical insomniac subgroups of interest, those with a physical comorbidity but not a psychiatric one, those with a psychiatric comorbidity but not a physical one, and those without either a physical or psychiatric comorbidity, large decrements in health utilities were observed for respondents who did not engage in any positive health behaviors $(0.61$, $0.57,0.64$, respectively) relative to good sleepers $(0.78)$. However, the gap in health utility scores between these subgroups and good sleepers diminishes with an increasing number of positive health behaviors (eg, clinical insomniacs with a physical comorbidity but not a psychiatric comorbidity performing all three positive health behaviors $=0.67$ vs good sleepers $=0.78$ ).

Discussion: A significant burden remains for those with insomnia who are treated. Given the particularly low levels of hrQOL among treated insomnia patients who have poor health behavior profiles and have psychiatric comorbidities, physicians should place particular emphasis on these patients who are most in need of intervention. Improved treatments may help to address the unmet needs of these patient populations.

Keywords: insomnia, quality of life, health behaviors

\section{Introduction}

Although its prevalence varies considerably by the definition used, ${ }^{1-8}$ insomnia is a serious condition with wide-ranging effects. Primary insomnia, as defined by the Diagnostic and Statistical Manual of Mental Disorders, fourth edition, Text Revision and the International Classification of Sleep Disorders, second edition by the American 
Academy of Sleep Medicine, is observed in approximately $6 \%$ of adults. ${ }^{2,3}$ However, symptoms of insomnia are quite common across the life cycle. ${ }^{8-10}$ For example, difficulty initiating sleep, difficulty maintaining sleep, and early morning awakening have been experienced in the past month by approximately $30 \%$ of the adult population. ${ }^{1,4,9,11}$

The presence of insomnia has been associated with increased anxiety and depression, ${ }^{12}$ impaired quality of life, ${ }^{12}$ and greater indirect and direct societal costs. ${ }^{13-17}$ Bolge et al used data from the 2005 United States National Health and Wellness Survey (NHWS) and found that respondents who had been diagnosed with insomnia and experienced their symptoms at least a few times per month reported significantly worse health-related quality of life (hrQOL), more missed work (absenteeism), more impairment while at work (presenteeism), and greater impairment in leisure activities. ${ }^{18}$ Indeed, a recent systematic review of 58 studies found a consistent effect of insomnia symptoms on social and work-related functioning, cognition and mood, and overall health care burden. ${ }^{19}$

However, despite this literature, fewer studies have focused on the burden of insomnia among patients in Japan. One exception is a recent study conducted by Sasai et al which categorized patients into "good sleepers" using and not using sleep medication and patients with insomnia using and not using sleep medication based on Pittsburgh Sleep Quality Index scores. ${ }^{20}$ The results suggested a significant physical and mental burden of insomnia for patients with insomnia in Japan and also suggested a further physical burden (but not a mental one) for those using medication, possibly due to the side effect profile of the medications.

The objective of the current study is to extend this work in Japan to further understand the effect of insomnia on hrQOL. More specifically, the analyses presented will examine the differences in health outcomes among those with insomnia and who are treated compared with those without insomnia or symptoms of insomnia. Further, another objective is to determine predictors of hrQOL among those who are using a medication for their insomnia to aid clinicians in the identification of potentially modifiable factors which can benefit the daily functioning of patients.

\section{Methods}

\section{Data source}

The current study used data from the 2012 Japan NHWS (Kantar Health, New York, NY, USA), an annual, cross-sectional study of adults aged 18 years or older $(\mathrm{N}=30,000)$. The NHWS is a general health survey which includes questions on medical conditions, symptoms, treatment information, and health outcomes among other variables. The survey is completed online and potential respondents to the NHWS are recruited through an existing Internet panel. The members of this panel are recruited through a variety of methods (eg, newsletters, online banner advertisements, etc). However, to minimize sampling bias, the NHWS recruited members of this panel using a stratified random sample framework (with quotas based on sex and age) to match the characteristics of the adult population in Japan. Comparisons between the Japan NHWS and governmental sources are reported elsewhere.

All respondents provided informed consent and the study protocol was reviewed and approved by an Institutional Review Board.

\section{Sample}

All respondents from the Japan NHWS were included in the analyses $(\mathrm{N}=30,000)$.

\section{Measures}

\section{Insomnia symptoms}

All respondents of the NHWS were asked whether they had insomnia or sleep difficulties (and, if so, whether those conditions had been diagnosed). All respondents were also presented with a list of sleep-related symptoms and asked to select which ones they regularly experience. These symptoms included "difficulty falling asleep", "waking during the night and not being able to get back to sleep", "poor quality of sleep", "waking up several times during the night", and "waking up too early".

Also, all respondents who reported they are experiencing insomnia or reported experiencing insomnia symptoms also indicated whether or not they are taking a medication for their condition. The specific medication was also reported by the respondent.

From these data, two groups were created to quantify the burden of insomnia: those who reported having been diagnosed with insomnia and using a prescription medication (clinical insomniacs) and those who did not report having insomnia or having symptoms associated with insomnia (good sleepers). We focused on clinical insomniacs to avoid including patients with a sub-clinical level of insomnia or those who were improperly managed (ie, above a clinical threshold for insomnia but not receiving adequate treatment).

\section{Demographics}

Demographic variables included sex, age, education (less than university graduate versus [vs] university graduate), 
annual household income ( $<¥ 3$ million $[\mathrm{MM}], ¥ 3 \mathrm{MM}$ to $<¥ 5 \mathrm{MM}, ¥ 5 \mathrm{MM}$ to $<¥ 8 \mathrm{MM}, ¥ 8 \mathrm{MM}$ or more, or decline to provide income), and employment status (currently employed, unemployed but looking for work [including those who are on disability], or unemployed but not looking for work [including those who are retired or homemakers]).

\section{Health history}

Smoking status ("do you currently smoke cigarettes?"; coded as current smoker [“yes"/“"yes, but I'm trying to quit"] vs current non-smoker ["never smoked"/“"no, I quit"/“no, I'm in the process of quitting"]), exercise behavior ("how many days in the past month did you exercise vigorously for at least $20 \mathrm{~min}$ utes?"; coded as exercised in the past month [1 day or more] vs did not exercise in the past month [0 days]), alcohol use ("how often do you drink alcohol?"; coded as currently drink alcohol [“daily"/“4-6 times per week"/“2-3 times per week"/“once a week"/“2-3 times per month"/“"once a month or less"] vs do not currently drink alcohol ["I do not drink alcohol"]), and body mass index. Physical comorbidities were assessed using the Charlson comorbidity index (CCI) which represents an index score summarizing the overall comorbidity burden of each respondent. The CCI is calculated by weighting the presence of severe comorbidities and summing the result. ${ }^{21}$ Patients who reported experiencing either anxiety or depression were considered to have a psychiatric comorbidity.

\section{hrQOL}

hrQOL was assessed using the physical component summary (PCS) and mental component summary (MCS) scores from the Short Form-36 version 2 (SF-36v2). ${ }^{22,23}$ The items from the SF-36v2 can also be used to calculate a health utility score; a score which quantitatively describes the overall health state of an individual. ${ }^{24}$ The health utility score varies from 0 (a health state equivalent to death) to 1 (a health state equivalent to perfect health). For example, based on the response pattern of the SF-36v2 questions (eg, a respondent reported their health as "excellent", they experienced no bodily pain, etc; all items from the SF-36v2), they are assigned a health utility score. The higher the health utility score, the better the overall health state of the respondent.

\section{Statistical analysis}

The results are presented in two sections. In the first section, demographic and health history differences among clinical insomniacs and good sleepers were examined using chi-square tests and one-way analysis of variance tests (ANOVAs). Effect sizes (Cohen's $d$ for continuous variables and $\phi$ coefficients for categorical variables) were also reported. Differences between these two groups with respect to hrQOL were then examined using multiple linear regressions controlling for age, sex, education, income, smoking, alcohol use, exercise, body mass index, and the CCI. Adjusted means from these regression models were reported.

The second section examined predictors of health utilities among clinical insomniacs using a multiple regression model. Predictors included demographics and health history variables. Unstandardized regression coefficients (b) and the $95 \%$ confidence intervals around those coefficients are provided. Adjusted means of different subgroups (eg, patients who exercise) were reported from the results of this model. Post hoc analyses then used the results of this regression model to understand how malleable behavioral factors (smoking, alcohol use, exercise) could potentially influence the health utility values among clinical insomniacs who either had a physical (based on the CCI) or psychiatric comorbidity burden. Among those with a physical comorbidity but not a psychiatric one, those with a psychiatric comorbidity but not a physical one, and those without either a physical or psychiatric comorbidity, the predicted health utility score was generated using the regression equation, assuming $0,1,2$, or 3 positive health behaviors (ie, no smoking, no alcohol use, regular exercise). These predicted health utility scores were then qualitatively compared to those without insomnia.

\section{Results}

\section{The burden of insomnia}

A total of $4.9 \%(n=1,455)$ of respondents reported a diagnosis of insomnia, with most $(70.0 \%$ using a prescription medication). These $n=1,018$ (3.4\% of the total adult population) participants who reported having insomnia and using a prescription treatment for their insomnia were defined as clinical insomniacs; $n=20,542$ were defined as good sleepers. The most common sleep symptoms experienced by clinical insomniacs included waking up several times at night (60.9\%), waking up too early (55.7\%), waking up and not getting back to sleep (52.9\%), and difficulty falling asleep (21.9\%). The demographic differences between these groups are shown in Table 1. Several differences were observed between clinical insomniacs and good sleepers. Specifically, clinical insomniacs were more likely to be female $(P<0.01)$, have a lower annual household income $(P<0.001)$, be unemployed but looking for work $(P<0.001)$, and less likely to be employed $(P<0.001)$. Clinical insomniacs were also more likely to smoke $(P<0.001)$, exercise regularly 
Table I Demographic and health history differences among clinical insomniacs and good sleepers

\begin{tabular}{|c|c|c|c|c|c|c|}
\hline & \multicolumn{2}{|c|}{$\begin{array}{l}\text { Clinical insomniacs } \\
(n=I, 0 \mid 8)\end{array}$} & \multicolumn{2}{|c|}{$\begin{array}{l}\text { Good sleepers } \\
(n=20,542)\end{array}$} & \multirow[t]{2}{*}{$P$-value } & \multirow[t]{2}{*}{ Cohen's d } \\
\hline & Mean & SD & Mean & SD & & \\
\hline Age & 47.9 & 14.7 & 47.0 & 15.8 & 0.080 & 0.28 \\
\hline \multirow[t]{2}{*}{$\mathrm{CCl}$} & 0.41 & 0.88 & 0.12 & $0.4 \mathrm{I}$ & 0.000 & 0.02 \\
\hline & $\%$ & $\mathbf{N}$ & $\%$ & $\mathbf{N}$ & $P$-value & $\phi$ \\
\hline Male & $46.7 \%$ & 475 & $50.9 \%$ & 10,448 & 0.009 & -0.02 \\
\hline University graduate & $44.5 \%$ & 453 & $47.0 \%$ & 9,650 & 0.081 & 0.02 \\
\hline Annual income: $<¥ 3 \mathrm{MM}$ & $24.2 \%$ & 246 & $17.8 \%$ & 3,666 & 0.000 & 0.04 \\
\hline Annual income: $¥ 3$ to $<¥ 5 \mathrm{MM}$ & $26.0 \%$ & 265 & $26.2 \%$ & 5,374 & & \\
\hline Annual income: $¥ 5$ to $<¥ 8 \mathrm{MM}$ & $23.1 \%$ & 235 & $25.7 \%$ & 5,288 & & \\
\hline Annual income: $¥ 8 \mathrm{MM}$ or more & $19.7 \%$ & 201 & $20.0 \%$ & 4,113 & & \\
\hline Annual income: decline & $7.0 \%$ & 71 & $10.2 \%$ & 2,101 & & \\
\hline Employed & $52.0 \%$ & 529 & $59.0 \%$ & 12,122 & 0.000 & 0.06 \\
\hline Not employed and looking for work & $7.1 \%$ & 72 & $2.7 \%$ & 552 & & \\
\hline Not employed and not looking for work & $41.0 \%$ & 417 & $38.3 \%$ & 7,868 & & \\
\hline Smoke & $34.9 \%$ & 355 & $20.2 \%$ & 4,145 & 0.000 & 0.08 \\
\hline Drink alcohol & $30.6 \%$ & 312 & $30.5 \%$ & 6,256 & 0.896 & 0.00 \\
\hline Regular exercise & $44.9 \%$ & 457 & $41.6 \%$ & 8,550 & 0.039 & 0.01 \\
\hline BMI: underweight & $15.9 \%$ & 162 & $10.7 \%$ & 2,199 & 0.000 & 0.06 \\
\hline BMI: normal weight & $59.3 \%$ & 604 & $68.7 \%$ & 14,119 & & \\
\hline BMI: obese & $22.3 \%$ & 227 & $16.3 \%$ & 3,344 & & \\
\hline BMI: unknown & $2.5 \%$ & 25 & $4.3 \%$ & 880 & & \\
\hline Psychiatric comorbidity & $51.5 \%$ & 524 & $2.2 \%$ & 459 & 0.000 & -0.51 \\
\hline Physical comorbidity & $26.9 \%$ & 274 & $9.2 \%$ & I,89| & 0.000 & -0.13 \\
\hline
\end{tabular}

Abbreviations: $\mathrm{CCl}$, Charlson comorbidity index; SD, standard deviation; BMI, body mass index; MM, million.

$(P<0.05)$, be obese $(P<0.001)$, and have a higher CCI score $(P<0.001)$.

The most common prescription medications used were zolpidem tartrate $(26.7 \%)$, brotizolam $(22.5 \%)$, flunitrazepam (21.2\%), and etizolam (19.1\%) (Table 2). A mean of 1.7 mediations were used by those with insomnia (standard deviation $=1.3$ ).

Adjusting for demographic and health history differences, clinical insomniacs reported significantly worse MCS and PCS scores relative to good sleepers (MCS: 34.2 vs 48.0, Cohen's $d=1.48$, respectively; PCS: 48.0 vs 52.8 , Cohen's $d=0.79$, respectively, both $P<0.001$ ) (Figure 1). Similarly, clinical insomniacs reported significantly worse health utility scores (0.61 vs 0.76 , Cohen's $d=1.21$, $P<0.001$; Figure 1).

\section{Predicting health status among those treated}

Among clinical insomniacs, an analysis of their health utility scores was examined (see Figure 2 for analyzed indices and Supplementary materials for complete regression tables). Employment status was the only demographic variable significantly associated with health utilities; those not employed and looking for work reported significantly lower health utilities $(\mathrm{b}=-0.035, P<0.05)$ than those employed (the reference group). Smoking $(\mathrm{b}=-0.016, P<0.05)$ and alcohol use $(b=-0.018, P<0.05)$ were associated with lower health utilities while exercise behavior was associated with higher health utility values $(b=0.023)(P<0.001)$. Although the CCI was significantly associated with lower health utility

Table 2 Prescription medication use among those with insomnia

\begin{tabular}{lll}
\hline & $\%$ & $\mathbf{N}$ \\
\hline Zolpidem tartrate & 26.7 & 272 \\
Brotizolam & 22.5 & 229 \\
Flunitrazepam & 21.2 & 216 \\
Etizolam & 19.1 & 194 \\
Triazolam & 17.4 & 177 \\
Alprazolam & 7.8 & 79 \\
Zopiclone & 7.3 & 74 \\
Nitrazepam & 6.3 & 64 \\
Estazolam & 4.9 & 50 \\
Trazodone hydrochloride & 4.6 & 47 \\
Lormetazepam & 3.9 & 40 \\
Rilmazafone hydrochloride & 3.3 & 34 \\
Quazepam & 2.8 & 28 \\
Flurazepam hydrochloride & 2.2 & 22 \\
Mirtazapine & 2.0 & 20 \\
Other & 12.9 & 131 \\
Number of medications used (mean, SD) & 1.7 & 1.3 \\
\hline
\end{tabular}

Abbreviation: SD, standard deviation. 


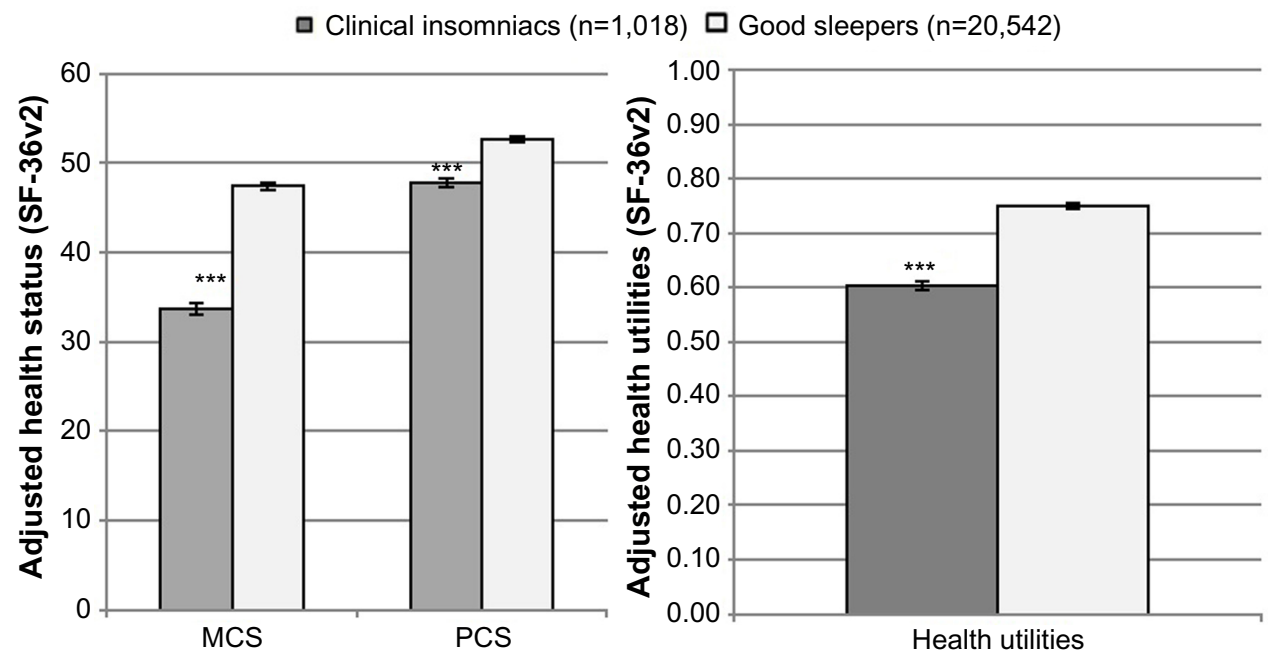

Figure I Adjusted means of health status measures (MCS and PCS scores from the SF-36v2) among clinical insomniacs versus good sleepers. Note: $* * * P<0.001$.

Abbreviations: SF, Short Form; MCS, mental component summary; PCS, physical component summary.

values $(b=-0.015)$, the strongest association was psychiatric comorbidities $(\mathrm{b}=-0.08)$ (both $P<0.001)$.

Using this regression model, subsequent analyses estimated the health utility scores for certain segments of clinical insomniacs. Specifically, health utility scores were estimated for respondents who had a varying number of positive health behaviors (from 0 to 3 of the following: do not smoke, do not drink alcohol, exercise regularly) among different physical and psychiatric comorbidity subgroups (Figure 3). For all three clinical insomniac subgroups of interest, those with a physical comorbidity but not a psychiatric one, those with a psychiatric comorbidity but not a physical one, and those without either a physical or psychiatric comorbidity, large decrements in health utilities were observed for respondents who did not engage in any positive health behaviors $(0.61$, $0.57,0.64$, respectively) relative to good sleepers (0.78). However, the gap in health utility scores between these subgroups and good sleepers diminishes with an increasing number of positive health behaviors. Indeed, for respondents with a physical comorbidity but not a psychiatric one and for respondents without either a physical or a psychiatric comorbidity who engage in all three positive health behaviors, the

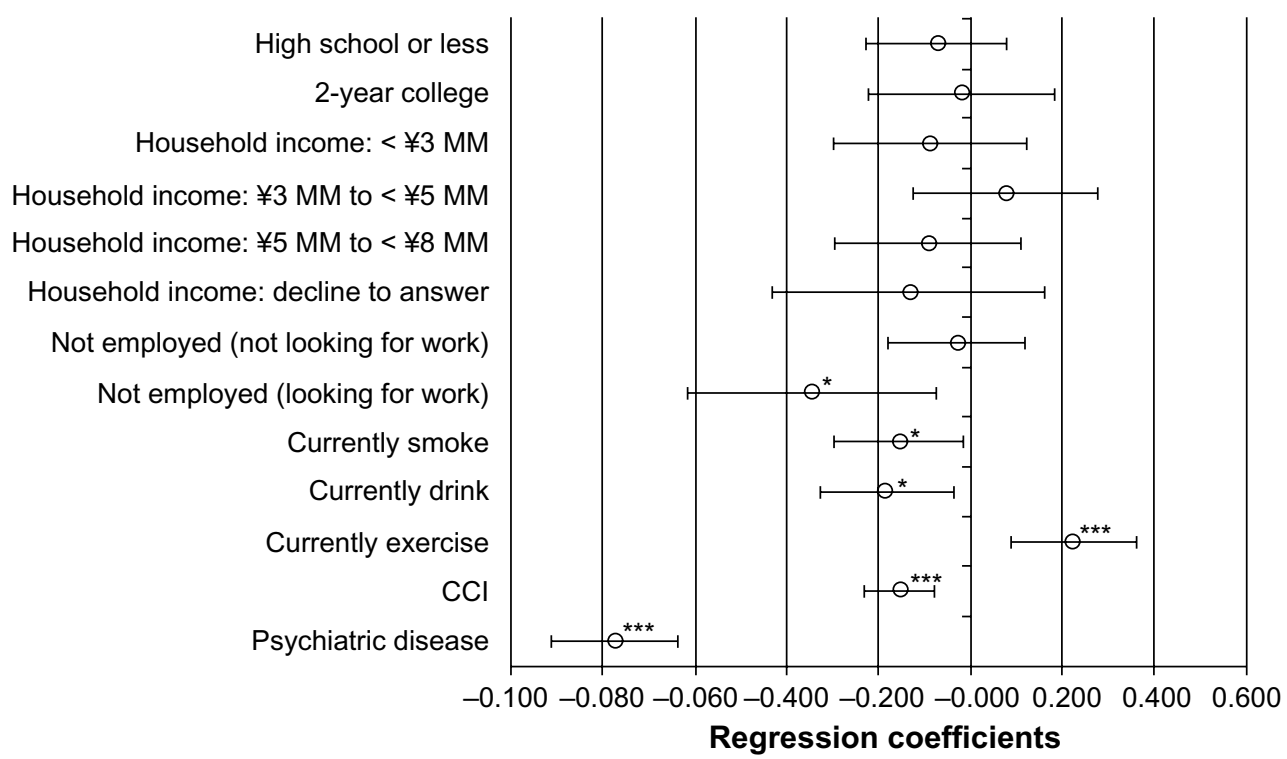

Figure 2 Unstandardized regression coefficients of predictors of health utility scores among clinical insomniacs.

Notes: All variables measured dichotomously with the exception of the $\mathrm{CCl}$. ${ }^{*} \mathrm{P}<0.05 ; * * \mathrm{P}<0.00 \mathrm{I}$. Bars represent $95 \%$ confidence intervals. Abbreviations: $\mathrm{CCl}$, Charlson comorbidity index; $\mathrm{MM}$, million. 


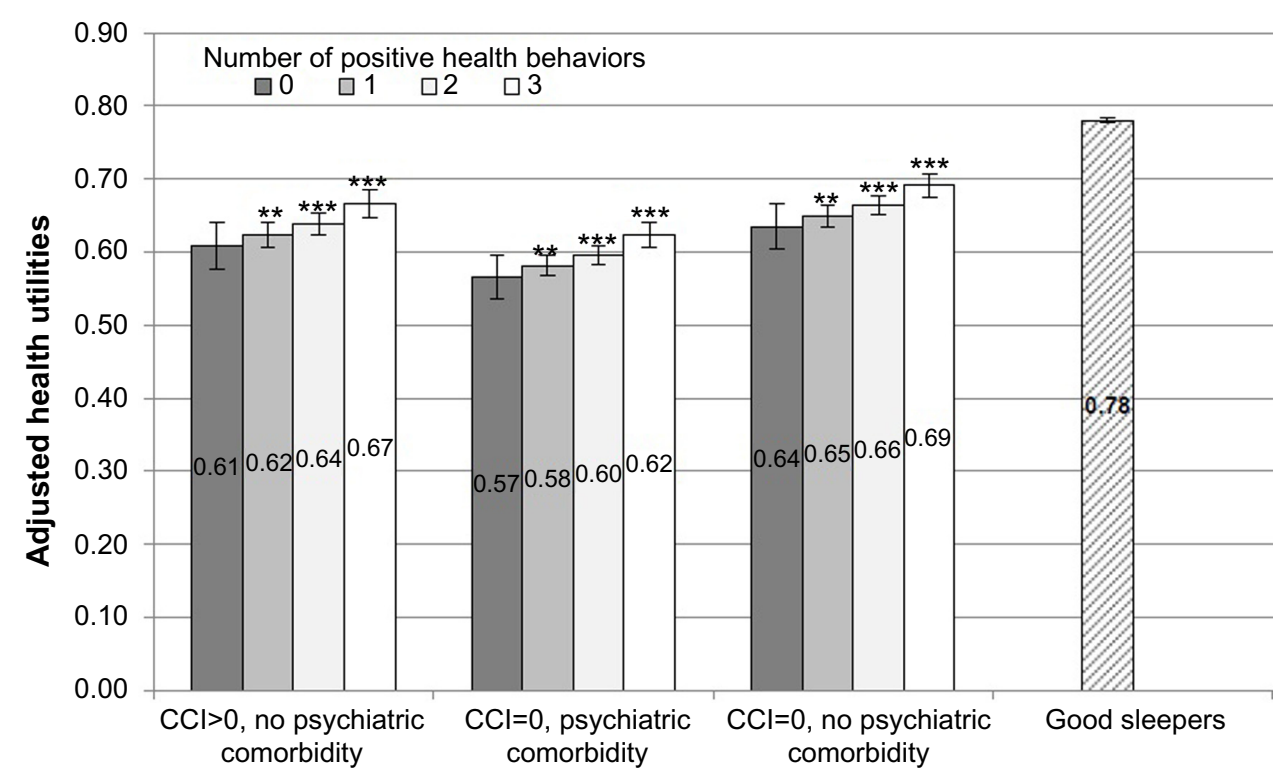

Figure 3 Predicted health utility scores for each clinical insomniac subgroup based on the number of positive health behaviors engaged in (smoking cessation, alcohol abstinence, and regular exercise) compared with good sleepers.

Notes: $* * P<0.0$ I; *** $P<0.00$ I relative to " 0 positive health behavior" category within each group (ie, those with a $\mathrm{CCl}>0$ and no $\mathrm{psychiatric} \mathrm{comorbidity;} \mathrm{those} \mathrm{with} \mathrm{CCl}$ $=0$ and a psychiatric comorbidity; those with $\mathrm{CCl}=0$ and no psychiatric comorbidity). The "good sleepers" bar was provided merely for context and was not part of the statistical model as they, by definition, did not have insomnia. Bars represent $95 \%$ confidence intervals.

Abbreviation: $\mathrm{CCl}$, Charlson comorbidity index.

gap in health utilities was reduced to 0.11 and 0.09 points, respectively ( 0.67 vs 0.78 and 0.69 vs 0.78 , respectively). However, due to the strong relationship between psychiatric comorbidities and health utilities, the gap between those with a psychiatric comorbidity still remained large despite the engagement in all three positive behaviors when compared with good sleepers (0.62 vs 0.78$)$.

\section{Discussion}

Although this study was not intended to be an epidemiological investigation of insomnia in Japan, our prevalence rate $(4.9 \%$ of the population was diagnosed; $3.4 \%$ of the adult population was diagnosed and treated) was generally consistent with the rates of primary insomnia. ${ }^{1-3}$ Naturally, our study does differ from the epidemiological studies which focused on defining insomnia purely through symptoms, ${ }^{6}$ as respondents who met criteria for a diagnosis of insomnia based on their symptom profile but have not been diagnosed would be excluded from our study but included in others. The discrepancy in prevalence observed between those who only report symptoms and those who are receiving a diagnosis (the latter further supported by the results here) suggests that a number of patients who meet criteria may not be receiving a diagnosis of insomnia.

It also should be noted that although there are undoubtedly cultural and health care system differences, the prevalence of insomnia and insomnia medication use was generally comparable between Japan and the West suggesting the potential generalizability of the findings. ${ }^{25-27}$

We observed a significant burden for patients with insomnia using a prescription medication (clinical insomniacs) compared with those without insomnia or insomnia symptoms (good sleepers) on hrQOL. This finding was similar to those reported in the United States ${ }^{18,19}$ and also extends the research by Sasai et al in Japan. ${ }^{20}$ Sasai et al compared differences across those with and without insomnia and those using and not using a medication. ${ }^{20}$ Although they had more analysis groups of interest, the authors found significant differences in MCS and PCS between clinical insomniacs and good sleepers, as reported here. ${ }^{6}$ However, our analyses controlled for a wider array of confounding variables (eg, CCI, health behaviors, etc) to provide further evidence of this effect. Despite the treatment they are receiving, clinical insomniacs still experience significant and clinically relevant decrements in hrQOL variables. These decrements are observed even after controlling for health history and comorbidity variables, reinforcing the effect insomnia has on patient functioning. This may, in part, be due to a lack of treatment benefit with respect to hrQOL. However, it is likely to be more than just a lack of effectiveness that is causing this gap. Certain sleep medications can be associated with dependency and residual effects, which could reduce levels of hrQOL. ${ }^{28}$ Further, although we controlled for both physical 
and mental comorbidities, sub-clinical levels of psychiatric conditions (perhaps more common in those with insomnia) ${ }^{29}$ may also contribute to a hrQOL decrements. Regardless of the reasons, a clear pattern of worse outcomes was observed for clinical insomniacs despite being under treatment.

The results also provide useful information as to what factors are associated with hrQOL among patients with insomnia, something not previously investigated. Specifically, behavioral factors (such as smoking and exercise) and comorbidity variables were among the strongest predictors of hrQOL among clinical insomniacs. Particularly, psychiatric comorbidities were the strongest predictors of all. Although a somewhat hypothetical exercise, the regression equation results suggest that through alleviating the effects of mental health comorbidities and promoting health behaviors related to sleep hygiene (smoking cessation, alcohol abstinence, and regular exercise), the health utility scores can approach that of good sleepers. Although we focused on malleable healthrelated factors, it is possible other variables may also be associated with health utilities (such as employment). These results have a number of clinical implications. Given past research has suggested that $40 \%$ of patients with insomnia have comorbid psychiatric illness, ${ }^{29}$ physicians should give particular emphasis to patients with poor behavioral profiles and psychiatric comorbidities as their hrQOL is likely to be poorest and most in need of intervention. Also, given the observed relationship between insomnia and an increased risk of depression, ${ }^{5}$ intervening even among clinical insomniacs with sub-clinical psychiatric symptoms may help to prevent future mood disorders in this population.

On the whole, these findings suggest that a combination of addressing the insomnia symptoms and taking mental health and behavior factors into consideration might maximize the hrQOL benefit to the patient. Similarly, these results suggest significant unmet needs with respect to insomnia treatments. A significant burden of insomnia remains even for those treated. The physical hrQOL (PCS) burden of insomnia reported in our current study was comparable (if not slightly larger) to that of diabetes while the mental burden was greater than diabetes, hypertension, obesity, and neuropathic pain underscoring the importance from a public health perspective of improved management. ${ }^{30,31}$ Aside from affecting patients' day-to-day functioning and hrQOL, insomnia has clear effects on society which could, potentially, be mitigated by optimizing treatments.

\section{Limitations}

All data were self-reported and no verification of an insomnia diagnosis or treatment usage was available. The NHWS did not include information on non-pharmacological treatment (eg, cognitive behavioral therapy, sleep routines, etc) which could be relevant to include in future studies. The study was cross-sectional so causality between insomnia, treatments, comorbidities, health behaviors, and hrQOL is only hypothesized. Although the NHWS is demographically representative, it is unclear the extent to which this analytical sample generalizes to the various insomnia subpopulations in Japan.

\section{Disclosure}

This study was funded by MSD KK, Japan. The authors have no other conflicts of interest to disclose.

\section{References}

1. Ohayon MM, Smirne S. Prevalence and consequences of insomnia disorders in the general population of Italy. Sleep Med. 2002;3(2): $115-120$.

2. American Psychiatric Association, editors. Diagnostic and Statistical Manual of Mental Disorders, Fourth Edition, Text Revision. Washington DC: American Psychiatric Association; 2000.

3. American Academy of Sleep Medicine, editors. The International Classification of Sleep Disorders. Diagnostic and Coding Manual. 2nd ed. Illinois: American Academy of Sleep Medicine; 2005.

4. Mellinger GD, Balter MB, Uhlenhuth EH. Insomnia and its treatment. Prevalence and correlates. Arch Gen Psychiatry. 1985;42(3):225-232.

5. Ford DE, Kamerow DB. Epidemiologic study of sleep disturbances and psychiatric disorders. An opportunity for prevention? JAMA. 1989; 262(11):1479-1484.

6. Kim K, Uchiyama M, Okawa M, Liu X, Ogihara R. An epidemiological study of insomnia among the Japanese general population. Sleep. 2000; 23(1):41-47.

7. Morin CM, LeBlanc M, Daley M, Gregoire JP, Mérette C. Epidemiology of insomnia: prevalence, self-help treatments, consultations, and determinants of help-seeking behaviors. Sleep Med. 2006;7(2):123-130.

8. Buysse DJ, Angst J, Gamma A, Ajdacic V, Eich D, Rössler W. Prevalence, course, and comorbidity of insomnia and depression in young adults. Sleep. 2008;31(4):473-480.

9. Hysing M, Pallesen S, Stormark KM, Lundervold AJ, Sivertsen B. Sleep patterns and insomnia among adolescents: a population-based study. J Sleep Res. 2013;22(5):549-556.

10. Kim WH, Kim BS, Kim SK, et al. Prevalence of insomnia and associated factors in a community sample of elderly individuals in South Korea. Int Psychogeriatr. 2013;25(10):1729-1737.

11. Furihata R, Uchiyama M, Takahashi S, et al. The association between sleep problems and perceived health status: A Japanese nationwide general population survey. Sleep Med. 2012;13(7):831-837.

12. Akashiba T, Kawahara S, Akahoshi T, et al. Relationship between quality of life and mood or depression in patients with severe obstructive sleep apnea syndrome. Chest. 2002;122(3):861-865.

13. Bonnet MH, Arand DL. 24-Hour metabolic rate in insomniacs and matched normal sleepers. Sleep. 1995;18(7):581-588.

14. Simon GE, VonKorff M. Prevalence, burden, and treatment of insomnia in primary care. Am J Psychiatry. 1997;154(10):1417-1423.

15. Sivertsen B, Overland S, Neckelmann D, et al. The long-term effect of insomnia on work disability: the HUNT-2 historical cohort study. Am J Epidemiol. 2006;163(11):1018-1024.

16. Ozminkowski RJ, Wang S, Walsh JK. The direct and indirect costs of untreated insomnia in adults in the United States. Sleep. 2007;30(3): 263-273. 
17. Daley M, Morin CM, LeBlanc M, et al. Insomnia and its relationship to health-care utilization, work absenteeism, productivity and accidents. Sleep Med. 2009;10(4):427-438.

18. Bolge SC, Doan JF, Kannan H, Baran RW. Association of insomnia with quality of life, work productivity, and activity impairment. Qual Life Res. 2009;18:415-422.

19. Ishak WW, Bagot K, Thomas S, et al. Quality of life in patients suffering from insomnia. Innov Clin Neurosci. 2012;9(10):13-26.

20. Sasai T, Inoue Y, Komada Y, Nomura T, Matsuura M, Matsushima E. Effects of insomnia and sleep medication on health-related quality of life. Sleep Med. 2010;11(5):452-527.

21. Liu GG, DiBonaventura MD, Yuan Y, et al. The burden of illness for patients with viral hepatitis C: evidence from a national survey in Japan. Value Health. 2012;15(Suppl 1):S65-S71.

22. Charlson ME, Pompei P, Ales KL, et al. A new method of classifying prognostic comorbidity in longitudinal studies: development and validation. J Chronic Dis. 1987;40(5):373-383.

23. Fukuhara S, Bito S, Green J, Hsiao A, Kurokawa K. Translation, adaptation, and validation of the SF-36 Health Survey for use in Japan. J Clin Epidemiol. 1998;51(11):1037-1044.

24. Fukuhara S, Ware JE Jr, Kosinski M, Wada S, Gandek B. Psychometric and clinical tests of validity of the Japanese SF-36 Health Survey. J Clin Epidemiol. 1998;51(11):1045-1053.
25. Brazier JE, Roberts J. The estimation of a preference-based measure of health from the SF-12. Med Care. 2004;42(9):851-859.

26. Ohayon M. Epidemiological study on insomnia in the general population. Sleep. 1996;19(3 Suppl 3):S7-S15.

27. Walsh JK, Ustun TB. Prevalence and health consequences of insomnia. Sleep. 1999;22:S427-S436.

28. Weyerer S, Dilling H. Prevalence and treatment of insomnia in the community: results from the Upper Bavarian Field Study. Sleep. 1991;14(5):392-398.

29. Frey DJ, Ortega JD, Wiseman C, Farley CT, Wright KP Jr. Influence of zolpidem and sleep inertia on balance and cognition during nighttime awakening: a randomized placebo-controlled trial. J Am Geriatr Soc. 2011;59(1):73-81.

30. Baglioni C, Battagliese G, Feige B, et al. Insomnia as a predictor of depression: a meta-analytic evaluation of longitudinal epidemiological studies. J Affect Disord. 2011;135(1-3):10-19.

31. DiBonaventura M, Fukuda T, Stankus AP. The prevalence and burden of comorbid hypertension and obesity among patients with type 2 diabetes in Japan. Presented at: ISPOR Asia-Pacific Conference; Sep 2012; Taipei, Taiwan. 


\section{Supplementary materials}

Table SI Regression results predicting mental component summary scores

\begin{tabular}{|c|c|c|c|c|c|}
\hline & b & SE & $95 \%$ LCL & $95 \%$ UCL & $\boldsymbol{P}$ \\
\hline Intercept & 42.369 & 0.856 & 40.691 & 44.047 & 0.000 \\
\hline Clinical insomniac & $-|3.72|$ & 0.270 & -14.250 & $-13.19 \mid$ & 0.000 \\
\hline Male & -0.007 & 0.133 & -0.266 & 0.253 & 0.960 \\
\hline Hokkaido & -1.035 & 0.743 & -2.492 & 0.422 & 0.164 \\
\hline Tohoku & -1.680 & 0.742 & -3.133 & -0.226 & 0.023 \\
\hline Kanto & -1.102 & 0.704 & -2.482 & 0.278 & 0.118 \\
\hline Chubu & -1.201 & 0.714 & -2.600 & 0.198 & 0.092 \\
\hline Kinki & -0.899 & 0.709 & -2.289 & 0.492 & 0.205 \\
\hline Chugoku & -0.776 & 0.742 & -2.231 & 0.680 & 0.296 \\
\hline Shikoku & -0.568 & 0.791 & -2.118 & 0.981 & 0.472 \\
\hline Kyushu & -0.389 & 0.728 & -1.816 & 1.038 & 0.593 \\
\hline Okinawa (reference) & - & - & - & - & - \\
\hline High school or less & -0.025 & 0.132 & -0.283 & 0.233 & 0.847 \\
\hline Two-year university & -0.032 & 0.172 & -0.370 & 0.306 & 0.852 \\
\hline Four-year university & - & - & - & - & - \\
\hline Annual income: $<¥ 3 \mathrm{MM}$ & -1.272 & 0.194 & -1.653 & -0.891 & 0.000 \\
\hline Annual income: $¥ 3$ to $<¥ 5 \mathrm{MM}$ & -0.465 & 0.172 & -0.802 & -0.128 & 0.007 \\
\hline Annual income: $¥ 5$ to $<¥ 8 \mathrm{MM}$ & -0.520 & 0.170 & -0.852 & -0.188 & 0.002 \\
\hline Annual income: $¥ 8 \mathrm{MM}$ or more & -1.045 & 0.227 & -1.490 & -0.600 & 0.000 \\
\hline Annual income: decline to answer (reference) & - & - & - & - & - \\
\hline Employed & 0.949 & 0.132 & 0.690 & 1.208 & 0.000 \\
\hline Not employed and looking for work & -1.709 & 0.347 & -2.389 & -1.028 & 0.000 \\
\hline Not employed and not looking for work (reference) & - & - & - & - & - \\
\hline National health insurance & 0.838 & 0.450 & -0.045 & 1.721 & 0.063 \\
\hline Social insurance & 1.165 & 0.449 & 0.285 & 2.046 & 0.009 \\
\hline Late stage elderly insurance & -1.256 & 0.657 & -2.543 & 0.031 & 0.056 \\
\hline None of the above & -1.493 & 0.534 & -2.538 & -0.447 & 0.005 \\
\hline Other insurance (reference) & - & - & - & - & - \\
\hline Current smoker & -0.406 & 0.144 & -0.689 & -0.124 & 0.005 \\
\hline Alcohol use & -0.359 & 0.128 & -0.609 & -0.109 & 0.005 \\
\hline Regular exercise & 1.510 & 0.117 & 1.281 & 1.740 & 0.000 \\
\hline BMI: underweight & -0.562 & 0.189 & -0.931 & -0.192 & 0.003 \\
\hline BMI: normal weight & -0.282 & 0.157 & -0.589 & 0.026 & 0.073 \\
\hline BMI: obese & -1.243 & 0.294 & -1.819 & -0.667 & 0.000 \\
\hline BMI: unknown (reference) & - & - & - & - & - \\
\hline Age & 0.154 & 0.004 & 0.146 & 0.162 & 0.000 \\
\hline $\mathrm{CCl}$ & -1.290 & 0.129 & -1.543 & -1.037 & 0.000 \\
\hline
\end{tabular}

Abbreviations: $\mathrm{CCl}$, Charlson comorbidity index; BMI, body mass index; SE, standard error, LCL, 95\% lower confidence level; UCL, $95 \%$ upper confidence level. 
Table S2 Regression results predicting physical component summary scores

\begin{tabular}{|c|c|c|c|c|c|}
\hline & b & SE & 95\% LCL & 95\% UCL & $P$ \\
\hline Intercept & 56.807 & 0.587 & 55.656 & 57.957 & 0.000 \\
\hline Clinical insomniac & -4.788 & 0.185 & -5.151 & -4.424 & 0.000 \\
\hline Male & 0.352 & 0.091 & 0.174 & 0.530 & 0.000 \\
\hline Hokkaido & -0.350 & 0.510 & -1.349 & 0.649 & 0.492 \\
\hline Tohoku & -0.452 & 0.508 & -1.449 & 0.545 & 0.374 \\
\hline Kanto & -0.109 & 0.483 & -1.056 & 0.837 & 0.821 \\
\hline Chubu & -0.079 & 0.489 & -1.039 & 0.880 & 0.871 \\
\hline Kinki & -0.188 & 0.486 & $-1.14 \mid$ & 0.765 & 0.699 \\
\hline Chugoku & -0.486 & 0.509 & -1.484 & 0.512 & 0.340 \\
\hline Shikoku & -0.325 & 0.542 & -1.388 & 0.737 & 0.548 \\
\hline Kyushu & 0.201 & 0.499 & -0.777 & 1.180 & 0.687 \\
\hline Okinawa (reference) & - & - & - & - & - \\
\hline High school or less & -0.230 & 0.090 & -0.407 & -0.053 & 0.011 \\
\hline Two-year university & -0.082 & 0.118 & -0.314 & 0.149 & 0.486 \\
\hline Four-year university & - & - & - & - & - \\
\hline Annual income: $<¥ 3 \mathrm{MM}$ & -0.460 & 0.133 & -0.721 & -0.199 & 0.001 \\
\hline Annual income: $¥ 3$ to $<¥ 5 \mathrm{MM}$ & -0.251 & 0.118 & -0.482 & -0.020 & 0.033 \\
\hline Annual income: $¥ 5$ to $<¥ 8 \mathrm{MM}$ & 0.008 & 0.116 & -0.220 & 0.235 & 0.949 \\
\hline Annual income: $¥ 8 \mathrm{MM}$ or more & 0.211 & 0.156 & -0.094 & 0.515 & 0.176 \\
\hline Annual income: decline to answer (reference) & - & - & - & - & - \\
\hline Employed & -0.290 & 0.091 & -0.468 & -0.113 & 0.001 \\
\hline Not employed and looking for work & -0.853 & 0.238 & -1.320 & -0.386 & 0.000 \\
\hline Not employed and not looking for work (reference) & - & - & - & - & - \\
\hline National health insurance & 0.851 & 0.309 & 0.246 & 1.457 & 0.006 \\
\hline Social insurance & 1.012 & 0.308 & 0.408 & 1.616 & 0.001 \\
\hline Late stage elderly insurance & -1.195 & 0.450 & -2.078 & -0.312 & 0.008 \\
\hline None of the above & -0.434 & 0.366 & $-|| 5 \mid$. & 0.283 & 0.235 \\
\hline Other insurance (reference) & - & - & - & - & - \\
\hline Current smoker & -0.262 & 0.099 & -0.456 & -0.068 & 0.008 \\
\hline Alcohol use & -0.361 & 0.087 & -0.532 & -0.189 & 0.000 \\
\hline Regular exercise & 1.046 & 0.080 & 0.888 & 1.203 & 0.000 \\
\hline BMI: underweight & -0.111 & 0.129 & -0.365 & 0.142 & 0.390 \\
\hline BMI: normal weight & -1.314 & 0.108 & -1.525 & -1.103 & 0.000 \\
\hline BMI: obese & -1.062 & 0.201 & -1.457 & -0.667 & 0.000 \\
\hline BMI: unknown (reference) & - & - & - & - & - \\
\hline Age & -0.063 & 0.003 & -0.068 & -0.057 & 0.000 \\
\hline $\mathrm{CCl}$ & -2.380 & 0.089 & -2.554 & -2.207 & 0.000 \\
\hline
\end{tabular}

Abbreviations: $\mathrm{CCl}$, Charlson comorbidity index; BMI, body mass index; SE, standard error, LCL, 95\% lower confidence level; UCL, $95 \%$ upper confidence level. 
Table S3 Regression results predicting health utilities

\begin{tabular}{|c|c|c|c|c|c|}
\hline & $\mathbf{b}$ & SE & $95 \%$ LCL & $95 \%$ UCL & $P$ \\
\hline Intercept & 0.751 & 0.012 & 0.728 & 0.774 & 0.000 \\
\hline Clinical insomniac & -0.146 & 0.004 & -0.153 & -0.139 & 0.000 \\
\hline Male & 0.009 & 0.002 & 0.005 & 0.012 & 0.000 \\
\hline Hokkaido & -0.004 & 0.010 & -0.024 & 0.016 & 0.722 \\
\hline Tohoku & -0.012 & 0.010 & -0.032 & 0.008 & 0.222 \\
\hline Kanto & -0.002 & 0.010 & -0.021 & 0.017 & 0.816 \\
\hline Chubu & -0.004 & 0.010 & -0.024 & 0.015 & 0.648 \\
\hline Kinki & -0.002 & 0.010 & -0.021 & 0.017 & 0.857 \\
\hline Chugoku & -0.004 & 0.010 & -0.024 & 0.016 & 0.694 \\
\hline Shikoku & -0.001 & 0.011 & -0.022 & 0.021 & 0.960 \\
\hline Kyushu & 0.006 & 0.010 & -0.014 & 0.025 & 0.567 \\
\hline Okinawa (reference) & - & - & - & - & - \\
\hline High school or less & -0.003 & 0.002 & -0.007 & 0.000 & 0.060 \\
\hline Two-year university & -0.001 & 0.002 & -0.006 & 0.003 & 0.564 \\
\hline Four-year university & - & - & - & - & - \\
\hline Annual income: $<¥ 3 \mathrm{MM}$ & -0.017 & 0.003 & -0.023 & -0.012 & 0.000 \\
\hline Annual income: $¥ 3$ to $<¥ 5 \mathrm{MM}$ & -0.009 & 0.002 & -0.013 & -0.004 & 0.000 \\
\hline Annual income: $¥ 5$ to $<¥ 8 \mathrm{MM}$ & -0.007 & 0.002 & -0.012 & -0.002 & 0.003 \\
\hline Annual income: $¥ 8 \mathrm{MM}$ or more & -0.010 & 0.003 & -0.016 & -0.004 & 0.002 \\
\hline Annual income: decline to answer (reference) & - & - & - & - & - \\
\hline Employed & 0.002 & 0.002 & -0.001 & 0.006 & 0.225 \\
\hline Not employed and looking for work & -0.021 & 0.005 & -0.030 & -0.011 & 0.000 \\
\hline Not employed and not looking for work (reference) & - & - & - & - & - \\
\hline National health insurance & 0.011 & 0.006 & -0.001 & 0.023 & 0.083 \\
\hline Social insurance & 0.017 & 0.006 & 0.005 & 0.029 & 0.006 \\
\hline Late stage elderly insurance & -0.033 & 0.009 & -0.051 & -0.015 & 0.000 \\
\hline None of the above & -0.021 & 0.007 & -0.036 & -0.007 & 0.004 \\
\hline Other insurance (reference) & - & - & - & - & - \\
\hline Current smoker & -0.005 & 0.002 & -0.009 & -0.002 & 0.006 \\
\hline Alcohol use & -0.004 & 0.002 & -0.007 & 0.000 & 0.040 \\
\hline Regular exercise & 0.018 & 0.002 & 0.015 & 0.021 & 0.000 \\
\hline BMI: underweight & -0.004 & 0.003 & -0.009 & 0.001 & 0.088 \\
\hline BMI: normal weight & -0.013 & 0.002 & -0.017 & -0.008 & 0.000 \\
\hline BMI: obese & -0.015 & 0.004 & -0.023 & -0.008 & 0.000 \\
\hline BMI: unknown (reference) & - & - & - & - & - \\
\hline Age & 0.001 & 0.000 & 0.000 & 0.001 & 0.000 \\
\hline $\mathrm{CCl}$ & -0.031 & 0.002 & -0.034 & -0.028 & 0.000 \\
\hline
\end{tabular}

Abbreviations: $\mathrm{CCl}$, Charlson comorbidity index; BMI, body mass index; SE, standard error, LCL, 95\% lower confidence level; UCL, 95\% upper confidence level.

\section{Publish your work in this journal}

Nature and Science of Sleep is an international, peer-reviewed, open access journal covering all aspects of sleep science and sleep medicine, including the neurophysiology and functions of sleep, the genetics of sleep, sleep and society, biological rhythms, dreaming, sleep disorders and therapy, and strategies to optimize healthy sleep. The journal welcomes original research, clinical \& epidemiological studies, reviews \& evaluations, case reports and extended reports. The manuscript management system is completely online and includes a very quick and fair peerreview system, which is all easy to use. Visit http://www.dovepress.com/ testimonials.php to read real quotes from published authors. 